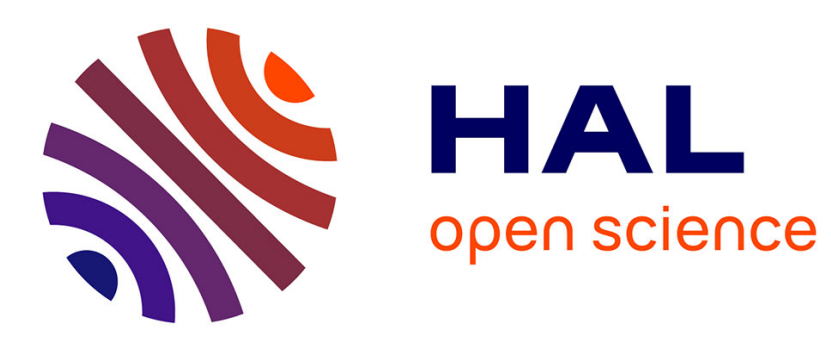

\title{
Electric field induced [MATH]-X transition in GaAs-AlAs coupled quantum well structures
}

\author{
M. Hagn, A. Zrenner, G. Böhm, G. Weimann
}

\section{To cite this version:}

M. Hagn, A. Zrenner, G. Böhm, G. Weimann. Electric field induced [MATH]-X transition in GaAsAlAs coupled quantum well structures. Journal de Physique IV Proceedings, 1993, 03 (C5), pp.C5229-C5-232. 10.1051/.jp4:1993543 . jpa-00251629

\section{HAL Id: jpa-00251629 https://hal.science/jpa-00251629}

Submitted on 1 Jan 1993

HAL is a multi-disciplinary open access archive for the deposit and dissemination of scientific research documents, whether they are published or not. The documents may come from teaching and research institutions in France or abroad, or from public or private research centers.
L'archive ouverte pluridisciplinaire HAL, est destinée au dépôt et à la diffusion de documents scientifiques de niveau recherche, publiés ou non, émanant des établissements d'enseignement et de recherche français ou étrangers, des laboratoires publics ou privés. 


\title{
Electric field induced $\Gamma$-X transition in GaAs-AlAs coupled quantum well structures
}

\author{
M. HAGN, A. ZRENNER, G. BÖHM and G. WEIMANN
}

Walter Schottky Institut, Technische Universität München, Am Coulombwall, 85748 Garching, Germany

\begin{abstract}
Phonon replicas are investigated at the electric field induced $\Gamma-\mathrm{X}$ transition in GaAs/AlAs coupled quantum well structures by photoluminescence spectroscopy. In the real- and k-space indirect regime the observed replicas are assigned to AlAs LO, TO and LA zone-edge phonons. At the indirect-direct transition those phonons disappear and a new replica emerges, which is assigned as a GaAs-LO phonon at the $\Gamma$-point. The observed phonon replicas are shown to be a local probe for the distribution of the ground state electronic wave function in the GaAs/AlAs coupled quantum well structure.
\end{abstract}

The absorption and recombination spectrum of a semiconductor material reflects directly the nature of the elementary band gap. Both direct and indirect gap semiconductors play important roles in optical device applications. Since the introduction of III-V quantum well (QW) structures the research activities in this field concentrated mainly on the excitonic properties of those structures and on QW lasers. For both topics direct gap systems such as GaAs/AlGaAs are favourable. Only in the field of GaAs/AlAs short period superlattices ${ }^{1}$ (SPSL) the indirect gap material AlAs attracted appreciable interest. As a function of the individual layer width the gap of a GaAs/AlAs SPSL can be tuned from direct to indirect 2 . Close to the critical layer thickness of the direct-indirect crossover the application of additional electric field ${ }^{3}$ or hydrostatic pressure 4 was show to result in the direct-indirect crossover $(\Gamma-X$ transition in the GaAs/AlAs system) as well. Recently a new GaAs/AlAs coupled QW (CQW) structure was introduced 5,6, which can be regarded as one period of a SPSL. In the present paper the electric field induced $\Gamma-X$ transition is studied in such a GaAs/AlAs CQW structure by means of photoluminescence (PL) spectroscopy.

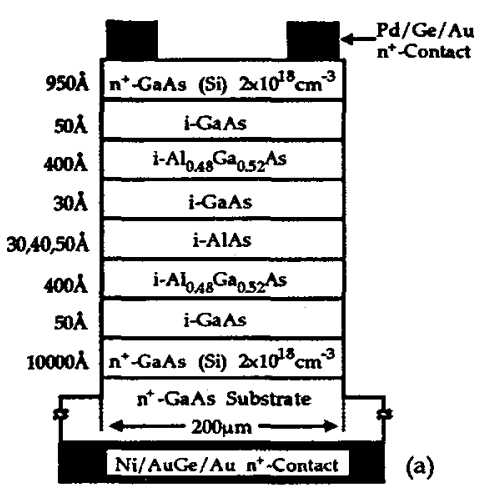

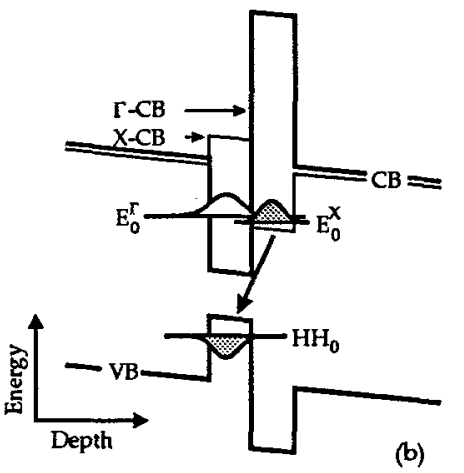

Fig. 1 (a) Layer sequence of the electric field tunable GaAs/AlAs CQW structure. (b) Band diagram of the active part of the structure for negative $V_{B}$. The realand $k$-space indirect transition $\mathrm{E}_{0} \mathbf{X} \rightarrow \mathrm{HH}_{0}$ is indicated. 
The investigated CQW structures are configured as electric field tunable hetero-type $n^{+}-i-n^{+}$photo diodes. The overall layer sequence and the band diagram of the active GaAs/AlAs CQW's in the center of the iregion are shown in Fig. 1. The CQW's are embedded between two $\mathrm{i}-\mathrm{Al}_{0.48} \mathrm{Ga}_{0.52} \mathrm{As}$ cladding layers. Electric tunability is obtained via the $n^{+}-G a A s$ layers on both sides of the intrinsic region. The top $\mathrm{n}^{+}-\mathrm{GaAs}$ layer is thin enough $(950 \AA)$ to allow for optical access to the active layers from the surface. The structures have been grown by molecular beam epitaxy (MBE). The GaAs QW width has been chosen $30 \AA$. For this width the confinement energy of the lowest electron subband $\mathrm{E}_{0} \Gamma(\approx 170 \mathrm{meV})$ compensates just about the offset between the GaAs $\Gamma$-conduction band (CB) and the AlAs X-CB ${ }^{6}$. Since the confinement energies for $X$-point subbands in the AlAs layer $E_{0} X$ are generally small $\left(m_{z z} \approx 1.1 \times m_{e}\right)$, those two subbands are energetically almost degenerate. Starting with this alignment the $\Gamma-X$ transition can be easily obtained by application of an external electric field. As indicated in Fig. 1, three different samples with an AlAs layer width of 30,40 and $50 \AA$ have been grown. Compared to a SPSL the present structure is asymmetric. As a consequence, the real- and $k$-space indirect transition from the $X$-point subband $E_{0} X$ in the AlAs layer is red-shifted for negative bias voltage $V_{B}$ and blue-shifted for positive $V_{B}$. In a SPSL there would be always a red-shift since there is an AlAs layer on either side of a given GaAs layer. In recent work on GaAs/AlAs $C Q W^{\prime}$ s the electric field induced $\Gamma-X$ transition was clearly observed ${ }^{6}$. The emission energies of the direct and indirect recombination as a function of $V_{B}$ could be described quantitatively.

In this contribution we analyse phonon assisted recombination channels to find information about the spatial location of the electronic ground state as a function of $V_{B}$. At the anticrossing this ground state undergoes a transition from the GaAs to the AlAs layer.

In the real- and $\mathbf{k}$-space indirect regime the recombination spectrum consists of an intense zero-phonon line and three phonon satellites. To identify the observed phonon replicas the observed emission lines are plotted relative to the energetic position of the zero phonon line for different $V_{B}$ in Fig. 2. The energy loss with respect to the zero phonon line is found to be $26.5,35.5$ and $48.5 \mathrm{meV}$. Almost similar numbers have been found previously in SPSL's 1,4 . In contrast to the previous work on SPSL's we assign part of those

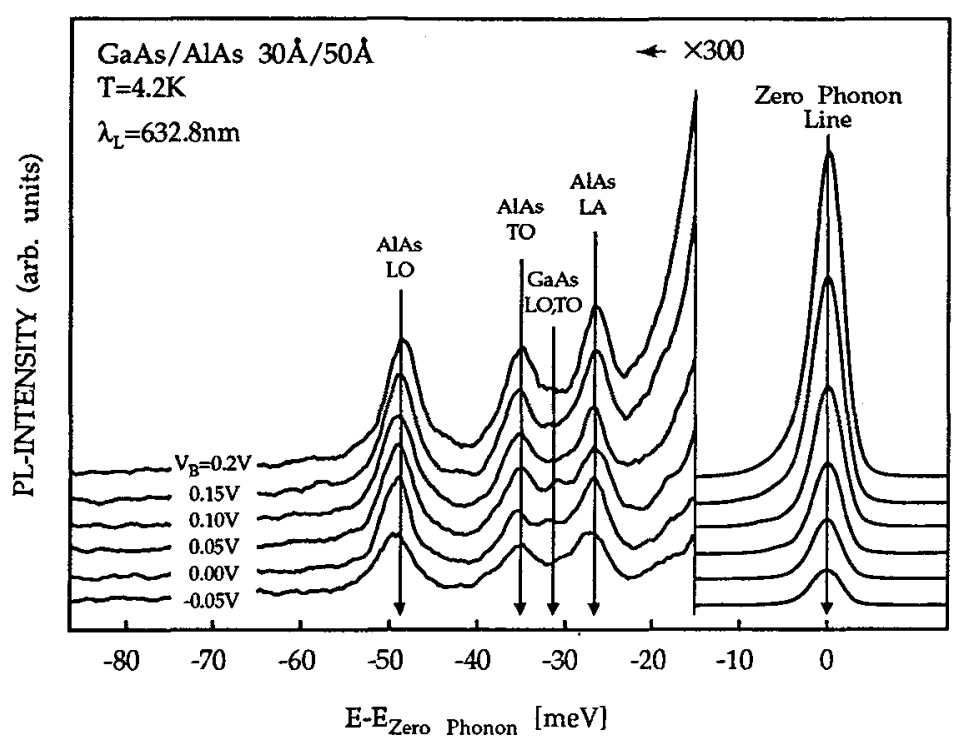

Fig. 2 X-point phonon replicas for various $V_{B}$ plotted relative to the energetic position of the zero phonon line. 


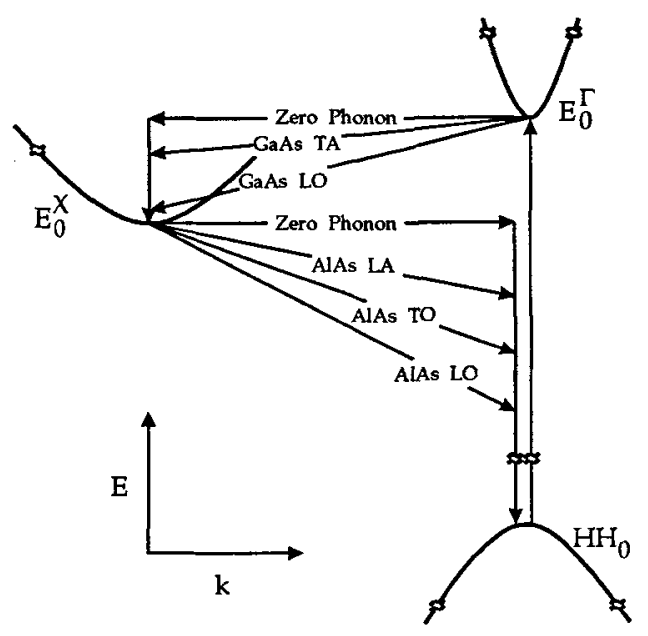

Fig. 3 Relevant channels of energy relaxation after optical excitation to $E_{0} \Gamma$. The energetic position of $E_{0} X$ can be tuned by $V_{B}$ with respect to $E_{0} \Gamma$

replicas to different phonons. This assignment is based on a comparison between a recent theoretical calculation of the phonon dispersions in GaAs and AlAs ${ }^{7}$ and our experimental data. Since the electronic ground state is localised in the AlAs layer at the X-point the emission of momentum conserving AlAs (and possibly GaAs) zone-edge phonons is expected. As shown in Fig. 2 we end up assigning the three observed replicas as AlAs LA, TO and LO X-point phonons. At the expected position of the GaAs LO and TO Xpoint replicas a clear emission can not be found. The main difference to previous assignments in SPSL's 1,4 is in the AlAs TO replica. This was previously thought to be a GaAs optical phonon replica. Since the dispersion of the AlAs TO phonon has been shown theoretically ${ }^{7}$ to be much stronger than previously believed, the observed emission can now be attributed to the X-point AlAs TO phonon. In summary we come to the conclusion that all observed replicas should originate from momentum conserving electronic transitions i.e. a scattering process from the AlAs X-point to a virtual state at the $\Gamma$-point with the emission of an AlAs-like zone-edge phonon. A schematic diagram of the electron relaxation after optical generation in the GaAs QW is shown in Fig. 3. The initial scattering process from $E_{0} \Gamma$ to $E_{0} X_{\text {is }}$ described in Ref. 5. In the following relaxation process from $\mathrm{E}_{0} \mathrm{X}$ to $\mathrm{HH}_{0}$ the optically observed transitions are indicated in accordance with the previous interpretations. For all three investigated CQW's with AlAs layer width of 30,40 and $50 \AA$ the energetic positions of the different phonon replicas were found to be the same. A possible contribution from confined phonons or interface modes is not observable in our experimental data.

Based on those arguments we can regard the phonon replicas as a local probe for the real-space position of the ground state electronic wave function in the GaAs/AlAs CQW. To further proof this interpretation we have analysed the evolution of the phonon replicas as a function of $V_{B}$ in the regime of the $\Gamma \cdot X$ transition. Results for the GaAs/AlAs $30 \AA / 30 \AA$ structure are shown in Fig. 4. In the indirect regime $\left(-1 V<V_{B}<0 V\right)$ the previously described AlAs-like replicas are observed. In the direct regime $\left(0.1 \mathrm{~V}<\mathrm{V}_{B}<1 \mathrm{~V}\right)$ those modes are absent. Instead a new mode appears which is interpreted as a $\Gamma$-point GaAs LO replica, in agreement with earlier work on SPSL's 4 . Right at the anticrossing at $V_{B}=0.1 \mathrm{~V}$ a cross-over between those two regimes is evident. This cross-over is mediated by the voltage-induced transfer of the ground state electron wave function from the AlAs to the GaAs QW. 


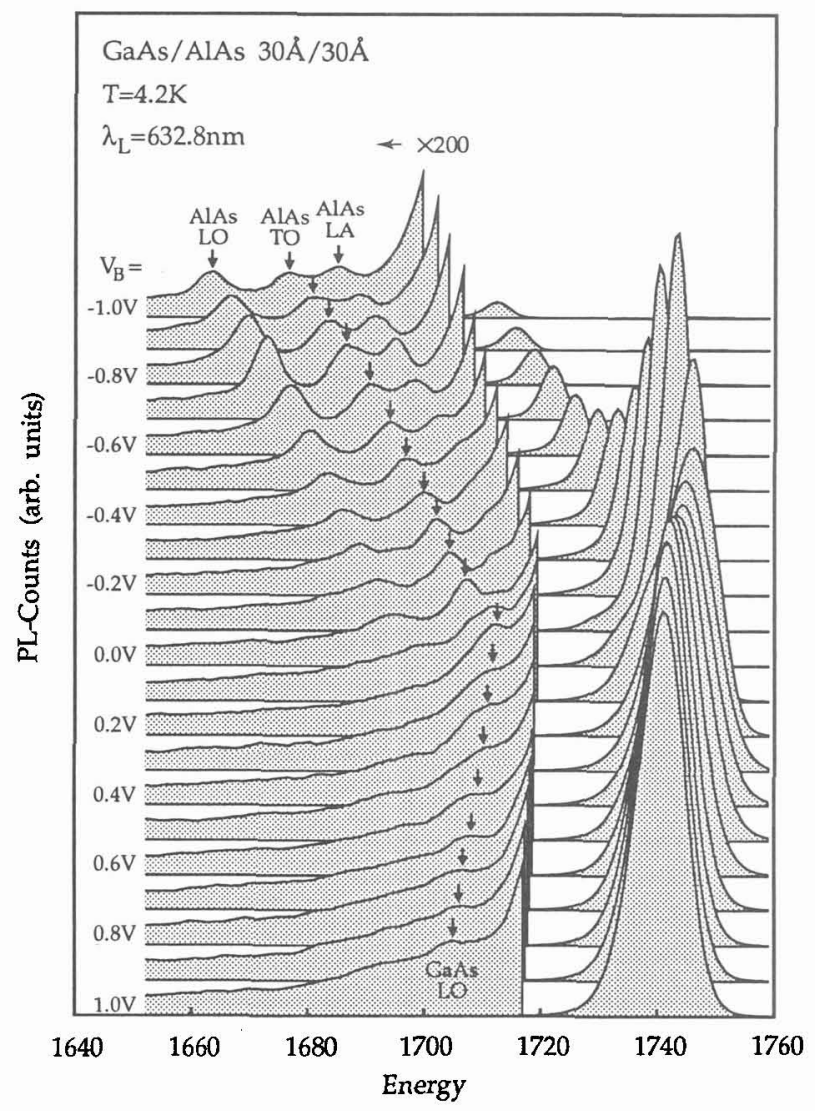

Fig. 4 Evolution of the phonon replicas as a function of $V_{B}$ in the regime of the $G$ $\mathrm{X}$ transition. The AlAs-like replicas at negative $V_{B}$ vanishes in the range of positive $V_{B}$ in favour of a GaAs-like replica.

The critical $V_{B}$ for this transfer cannot be determined reliably by PL or PL-excitation spectroscopy because of the inherent changes in exciton lifetime and line shape or absorption. The spectroscopy of the phonon replicas however allows further insights into the properties of the electronic ground state in this CQW system. The phonon replicas provide thereby a local probe of the phonon spectrum in the QW of interest, namely in the QW in which the electronic ground state is localised. This work was financially supported by the BMFT Photonics project.

[1] Finkman E., Sturge M. D. and Tamargo M. C., Appl. Phys. Lett. 49, 1299 (1986)

[2] Moore K. J., Duggan G., Dawson P. and Foxon C. T., Phys. Phys. B 38, 5535 (1988)

[3] Meynadier M. H., Nahory R. E., Worlock J. M., Tamargo M. C., Miguel J. L. and Sturge M. D., Phys. Rev. Lett. 60, 1338 (1988)

[4] Skolnick M. S.,. Smith G. W., Spain I. L., Whitehouse R. C., Herbert D. C., Whittaker D. M. and Reed L. J., Phys. Rev. B 39, 11191 (1989)

[5] Zrenner A., Leeb P., Schäfer J., Böhm G., Weimann G., Worlock J. M., Florez L. T. and Harbison J. P., Surf. Sci. 263, 496 (1992)

[6] Zrenner A., Festkörperprobleme/Advances in Solid State Physics, Vol. 32 (ed. by U. Rössler, Vieweg, Braunschweig/Wiesbaden 1992), pp. 61-80.

[7] Fasolino A., Molinari E. and Kunc K., Phys. Rev. B 41, 8302 (1990) 